\title{
The current status of drug-coated balloons in in-stent restenosis treatment
}

\author{
Leszek Bryniarski, Łukasz Klima, Sławomir Surowiec \\ Department of Cardiology, Interventional Electrophysiology and Hypertension, University Hospital, Jagiellonian \\ University Medical College, Kraków, Poland
}
Abstract Restenosis after angioplasty has always been a significant problem in interventional cardiology and is associated with significant morbidity and costs, with a wide spectrum of clinical presentation. The population treated with bare metal stents and drug eluting stents is very large and because of the complex subset of patients and lesions the overall rate of in-stent restenosis remains significant. For interventional cardiologist several treatment options are available nowadays such as repeat stenting, balloon angioplasty, drug-coated balloons but due to lack of precise guidelines the man- agement remains challenging. In this paper authors provide comprehensive review of novel tech- nique in in stent restenosis treatment: drug eluting balloons.

Key words in-stent restenosis, drug eluting balloons, drug coated balloons.

\section{Introduction}

$\mathbf{P}$ ercutaneus coronary interventions (PCI) has evolved to the mainstream revascularization method far outnumbering coronary artery bypass grafting (CABG). However, the major drawback of $\mathrm{PCl}$ is a higher rate of target lesion revascularization (TLR) in comparison to CABG due to in-stent restenosis. Restenosis after angioplasty has always been a significant problem in interventional cardiology and is associated with significant morbidity and costs, with a wide spectrum of clinical presentation. The disadvantages of balloon angioplasty has been removed when bare metal stents (BMS) were invented. The introduction of these devices significantly reduced restenosis after angioplasty. Development of drug eluting stents (DES) an another milestone in treatment of patients with coronary artery disease. The high efficacy of these devices in prevention of restenosis compared with bare metal stents has allowed percutaneus coronary intervention to be used in complex lesions. Introduction of DES reduced the number of target lesion revascularizations but did not eliminate the in-stent restenosis (ISR). The population treated with BMS and DES is very large ${ }^{1}$ and because of the complex subset of patients and lesions the overall rate of ISR remains significant. Although several treatment options are available nowadays such as repeat stenting, balloon angioplasty (BA), drug-coated balloons (DCB) there it is always a dilemma for interventional cardiologist which devices to use, so management remains challenging. We provide comprehensive review of novel technique in ISR treatment: drug eluting balloons (DEBs).

\section{Definition}

Restenosis is defined as reduction of $\geq 50 \%$ of lumen diameter after $\mathrm{PCl}$ at follow-up angiography. Although this definition was used as end point in large number of clinical trials it has to be emphasized that apart from angiographic findings clinical manifestation of ischemia plays significant role in decision of treatment.

\section{Pathophysiology}

After stent implantation, which damages the endothelium and leads to regional and systemic inflammatory reaction, process of neointima proliferation begins and in months leads to neointimal hyperplasia ${ }^{2}$. Stent underexpansion or not optimal atherosclerotic plaque coverage with stent favors ISR. But according to the latest findings it seems that one more factor is responsible for ISR, especially in late stage - neoatherosclerosis ${ }^{3}$. What is interesting novel atherosclerotic plaques are formed faster in drug eluting stent (after one year) than in bare metal stent (after four years). Detailed description of pathophysiology of ISR is will not be the subjects of this paper. Type of ISR and cause of its occurrence determines its treatment.

\section{Treatment}

When treating ISR we should take several possibilities into consideration and these are: optimal medical therapy (OMT), balloon angioplasty, stenting (BMS, the same $D E S$, different DES), DEB and cardiac surgery. In first human trial on DEB - a PACCOCATH ISR I/II patients with BMS-ISR were randomized to Paccocath DEB versus standard balloon angioplasty. After 6 months major cardiac 
events (MACE) appeared in 4\% in DEB arm and 31\% in BA group ${ }^{4}$. After 24 months observation MACE rated $11 \%$ vs $46 \%$ and target lesion revascularization (TLR) $6 \%$ and $37 \%$ respectively in $\mathrm{DEB}$ and $\mathrm{BA} \mathrm{arm}^{5}$. In other trial on drugcoated balloons use of DEB resulted in smaller late lumen loss (LLL) in BMS restenosis to compare with DES restenosis $(-0,05$ vs $0,19 \mathrm{~mm}$ respectively) what reflected in fewer revascularizations at one year ( 2.4 vs $17.1 \%$ respectively) ${ }^{6}$. Similarly, both the Spanish DIOR and the SeQuent Please registries ${ }^{7,8}$ highlighted better clinical outcomes with BMS ISR compared to DES ISR. However, outcomes of paclitaxel versus non-paclitaxel DES ISR treated with Sequent Please did not differ (TLR: $8.3 \%$ versus 10.8\%; $\mathrm{P}=0.46$ ). Perhaps the efficacy of drug coated balloons in treating BMS ISR reflects the fact that a drug is introduced for the first time, whereas DCB is less efficacious in a 'drug-resistant' vessel manifesting DES ISR. Another reason could be that drug eluting stents are implanted in complex lesions which predisposes to restenosis. Comparison of drug-coated balloons with drug eluting stents (SeQuent Please, B. Braun versus TAXUS stent by Boston Scientific) in management of in-BMS restenosis was analyzed in PEPCAD II trial which demonstrated non-inferiority. After 6 months of follow up late lumen loss was estimated as $0,38 \pm 0,61 \mathrm{~mm}$ in DES and 0,17 $\pm 0,42 \mathrm{~mm}$ in DEB $(P=0,03)$ and binary restenosis respectively $20 \%$ i $7 \%$ $(P=0,03)$. After 12 months of observation MACE appeared in $22 \%$ in $D E S$ and $9 \%$ in DEB group $(P=0,08)^{9}$. In recently published German consensus on drug-coated balloon [10] authors suggest to treat BMS in-stent restenosis with drug-coated balloons leaving DES as a bailout therapy when dissection after predilatation appears with TIMI flow < III or when residual stenosis exceeds $30 \%$. The used DEB should extend 2-3 $\mathrm{mm}$ beyond the pre-dilated area, balloon/vessel ratio should equal 0.8-1.0 and pressure 8-10 atm, whereas time of inflation must not be shorter than 30 second. The authors suggest predilatation with conventional balloon not longer than stent if ISR is restricted to stent, with diameter approximately 0.5 $\mathrm{mm}$ smaller than vessel diameter and with nominal pressure. Second conventional balloon should be shorter than stent with balloon to vessel diameter ratio 0.8-1.0 and inflated with pressure 12-16 atm. The European guidelines give DEB class Ila and level of evidence $B$ in bare metal stent restenosis treatment ${ }^{11}$.

In DES ISR situation is more complicated and there is now clear evidence what kind of management gives the best results. There is evidence that implantation of another DES is better than BA ${ }^{12}$. ISAR DESIRE 2 study was designed to answer the question whether we should use stent eluting the same or alternative drug. There was no difference between sirolimus eluting stent (SES) or paclitaxel eluting stent (PES) in LLL, TLR or binary restenosis ${ }^{13}$. In other trial, PEPCAD-DES ${ }^{14}$, where DEB and $B A$ in treatment DES ISR were compared the TLR and MACE rated significantly lower in group of drug-coated balloon ( $16.7 \%$ vs $50 \%$ and $15.3 \%$ vs $36,8 \%$ respectively). The treatment with DEB resulted with significantly lower LLL (0.43 versus $1.03 \mathrm{~mm}$ ) and restenosis (17.2\% versus $58.1 \%$ ). Finally in recent ISAR DESIRE 3 study ${ }^{15}$ the comparison of all three methods (BA, DEB, DES) in treatment of DES in-stent restenosis was made. There was no difference between diameter stenosis between DEB and DES (38\% and $37.4 \%$ respectively) and both methods were superior to BA (54.1\%). In terms of binary stenosis DEB and DES were comparable $(26.5 \%$ and $24.0 \%)$ and superior to BA (56.7\%). As for TLR in DEB $22.1 \%$, in DES $13.5 \%(P=0.09)$, in $B A 43.5 \%$. That led authors to conclusion that in patients presenting with ' $\mathrm{li}$ mus'-DES restenosis, DEB therapy is non-inferior to repeat stenting with PES. Both DEB and PES therapy are superior to balloon angioplasty alone.

It may seem counterintuitive that short balloon inflation with DEB can be superior to DES which have a longer duration of drug but delivery there are several theoretical advantages of DEB:

- avoiding the problem of permanent implant causing inflammation and hypersensitivity reaction

- delivery of antiproliferative medication when needed, for instance immediately after the barotraumas induced by balloon angioplasty

- avoiding multiple layers of stent

- avoiding the potential risk of corrosion of the stent: the mechanical friction between overlapping stents and chemical reactions between dissimilar alloys if mixing different stent types could lead to corrosion. Stent alloys form a protective oxide film, insulating the stent struts from the corrosive body fluids. There is a risk of mechanical damage of the oxide film caused micromotion at point of stent overlap. If this protective film is getting scratched off by overlapping stent struts the underlying stent struts get exposed and may undergo corrosion.

- overlapping stents of different alloys could theoretically lead to galvanic corrosion. This can be avoided by DEB. However, these concerns are rather theoretical, there is no clinical evidence which indicates a problem of using different types of overlapping stents ${ }^{16}$.

\section{Conclusion}

By obviating the need for additional stent implantation treatment with a drug-eluting balloon may be the treatment of choice in patients presenting with 'limus'-DES restenosis. In bare metal stent restenosis the drug-coated balloon should be the golden standard therapy leaving DES implantation only as bailout therapy. Balloon angioplasty can be successfully used when stent underexpansion diagnosed with IVUS is cause of ISR and when dealing with focal restenosis.

\section{References}

1. Cutlip DE, Chauhan MS, Baim DS, et al. Clinical restenosis after coronary stenting: perspectives from multicenter clinical trials. J Am Coll Cardiol 2002; 40(12):2082-9.

2. Dangas GD, Claessen BE, Caixeta A, et al. In-stent restenosis in the drug-eluting stent era. J Am Coll Cardiol 2010; 56(23): 1897-907.

3. Park SJ, Kang SJ, Virmani R, et al. In-stent neoatherosclerosis: a final common pathway of late stent failure. J Am Coll Cardiol 2012; 59(23): 2051-7.

4. Scheller B, Hehrlein C, Bocksch W, et al. Treatment of coronary in-stent restenosis with a paclitaxel-coated balloon catheter. $\mathrm{N}$ Engl J Med 2006; 355(20): 2113-24. 
5. Scheller B, Hehrlein C, Bocksch W, et al. Two year follow-up after treatment of coronary in-stent restenosis with a paclitaxel-coated balloon catheter. Clin Res Cardiol 2008; 97(10): 773-81.

6. Hehrlein C, Dietz U, Kubica J, et al. Twelve-month results of a paclitaxel releasing balloon in patients presenting with in-stent restenosis First-in-Man (PEPPER) trial. Cardiovasc Revasc Med 2012; 13(5): 260-4.

7. Wohrle J, Zadura M, Mobius-Winkler S, et al. SeQuentPlease World Wide Registry: clinical results of SeQuent please paclitaxel-coated balloon angioplasty in a large-scale, prospective registry study. J Am Coll Cardiol 2012; 60(18): 1733-8.

8. Vaquerizo B, Serra A, Miranda-Guardiola F, et al. One-year outcomes with angiographic follow-up of paclitaxel-eluting balloon for the treatment of in-stent restenosis: insights from Spanish multicenter registry. J Interv Cardiol 2011; 24(6): 518-28.

9. Rosenkranz S, Maier LS, Maack C, et al. Hotline update of clinical trials and registries presented at the German Cardiac Society Meeting 2007: 2L-Registry, Kardio-Pro, EVER, AFFECT, VTACH, ARTS II, OPTAMI, PEPCAD I, PEPCAD II, GERSHWIN, SPICE, FIXCHF and CREDIT. Clin Res Cardiol 2007; 96(7): 457-68.

10. Kleber FX, Mathey DG, Rittger $\mathrm{H}$, et al. How to use the drugeluting balloon: recommendations by the German consensus group. Eurolntervention 2011; 7 Suppl K: K125-8.
11. Wijns W, Kolh P, Danchin N, et al. Guidelines on myocardial revascularization. Eur Heart J 2010; 31(20): 2501-55.

12. Song HG, Park, DW, Kim YH, et al. Randomized trial of optimal treatment strategies for in-stent restenosis after drug-eluting stent implantation. J Am Coll Cardiol 2012; 59(12): 1093-100.

13. Mehilli J, Byrne RA, Tiroch K, et al. Randomized trial of paclitaxelversus sirolimus-eluting stents for treatment of coronary restenosis in sirolimus-eluting stents: the ISAR-DESIRE 2 (Intracoronary Stenting and Angiographic Results: Drug Eluting Stents for In-Stent Restenosis 2) study. J Am Coll Cardiol 2010; 55(24): 2710-6.

14. Rittger H, Brachmann J, Sinha AM, et al. A randomized, multicenter, single-blinded trial comparing paclitaxel-coated balloon angioplasty with plain balloon angioplasty in drug-eluting stent restenosis: the PEPCAD-DES study. J Am Coll Cardiol 2012; 59(15): 1377-82.

15. Byrne RA, Neumann FJ, Mehilli J, et al. Paclitaxel-eluting balloons, paclitaxel-eluting stents, and balloon angioplasty in patients with restenosis after implantation of a drug-eluting stent (ISAR-DESIRE 3): a randomised, open-label trial. Lancet 2013; 381(9865): 461-7.

16. Indermuehle A, Bahl R, Lansky AJ, et al. Drug-eluting balloon angioplasty for in-stent restenosis: a systematic review and meta-analysis of randomised controlled trials. Heart 2013; 99(5): 327-33. 